\title{
Concentração de íons na solução de um Latossolo Vermelho sob diferentes sistemas de manejo
}

\author{
Maria Inês Lopes de Oliveira(1), Thierry Becquer(2), Wenceslau J. Goedert( ${ }^{(3)}$, \\ Lourival Vilela(4) e Philippe Deleporte(5)
}

\begin{abstract}
(1)Universidade Estadual de Londrina, Departamento de Agronomia, Rodovia Celso Garcia Cid, Caixa Postal 6001, CEP 86051-970 Londrina, PR. E-mail: minesoliveira@yahoo.com.br ${ }^{(2)}$ Institut de Recherche pour le Développement, UMR Eco\&Sols, 2 Place Viala, F-34060 Montpellier Cedex 02, France. E-mail: thierry.becquer@ird.fr (3)Universidade de Brasília, Departamento de Agronomia, Caixa Postal 04508, CEP 70710-900 Brasília, DF. E-mail: goedert@unb.br ${ }^{(4)}$ Embrapa Cerrados, BR-020 Km 18, Caixa Postal 08223, CEP 73301-970 Planaltina, DF. E-mail: Ivilela@cpac.embrapa.br ${ }^{(5)}$ Centre de Coopération Internationale en Recherche Agronomique pour le Développement, UMR Eco\&Sols, 2 Place Viala, F-34060 Montpellier Cedex 02, France. E-mail: philippe.deleporte@cirad.fr
\end{abstract}

Resumo - O objetivo deste trabalho foi avaliar as concentrações de íons na solução de um Latossolo Vermelho, em diferentes sistemas de manejo. Foram avaliados os sistemas de lavoura e pastagem contínua, assim como os rotacionados em integração lavoura-pecuária. O experimento foi iniciado em 1991, e as coletas das soluções foram realizadas em 2005 e 2006. Cápsulas porosas foram instaladas às profundidades de $20 \mathrm{e} 150 \mathrm{~cm}$, e as soluções foram extraídas em seis épocas de cada ano. As concentrações de $\mathrm{Cl}^{-}, \mathrm{SO}_{4}{ }^{2-}, \mathrm{NO}_{3}^{-}, \mathrm{H}_{2} \mathrm{PO}_{4}^{-}, \mathrm{K}^{+}, \mathrm{Mg}^{2+}$ e $\mathrm{Ca}^{2+}$ foram determinadas. Independentemente das profundidades e dos sistemas de manejo, a concentração de íons nas soluções, em ordem decrescente de grandeza, foi: $\mathrm{NO}_{3}{ }^{-}>\mathrm{Cl}^{-}>\mathrm{SO}_{4}{ }^{2-}>\mathrm{H}_{2} \mathrm{PO}_{4}{ }^{-}$e $\mathrm{Ca}^{2+}>\mathrm{K}^{+}>\mathrm{Mg}^{2+}$. Entre os sistemas de cultivo, a concentração dos íons na solução decresceu e foi maior em lavoura contínua sob preparo convencional, seguida de lavoura contínua sob plantio direto e, finalmente, pelo sistema de integração lavoura-pecuária e pela pastagem contínua. À profundidade de $150 \mathrm{~cm}$, as concentrações dos íons na solução do solo sob pastagem contínua e integração lavoura-pecuária foram sempre baixas, o que indica baixo risco de lixiviação.

Termos para indexação: Cerrado, integração lavoura-pecuária, lixiviação, pastagem, solo.

\section{Ion concentrations in the solution of an Oxisol under different management systems}

\begin{abstract}
The objective of this work was to evaluate ion concentrations in an Oxisol solution, in plots subjected to different management systems. Continuous crop and pasture, and the integrated crop-livestock management system were evaluated. The experiment started in 1991, and the soil solutions were collected in 2005 and 2006. Ceramic cups were installed at 20 and $150 \mathrm{~cm}$ soil depths, and the solutions were extracted six times each year. The concentrations of $\mathrm{Cl}^{-}, \mathrm{SO}_{4}{ }^{2-}, \mathrm{NO}_{3}{ }^{-}, \mathrm{H}_{2} \mathrm{PO}_{4}^{-}, \mathrm{K}^{+}, \mathrm{Mg}^{2+}$, and $\mathrm{Ca}^{2+}$ were determined. Irrespective of the depths and cropping systems considered, the concentrations of ions into the soil solutions, in decreasing order, were: $\mathrm{NO}_{3}{ }^{-}>\mathrm{Cl}^{-}>\mathrm{SO}_{4}{ }^{2-}>\mathrm{H}_{2} \mathrm{PO}_{4}{ }^{-}$and $\mathrm{Ca}^{2+}>\mathrm{K}^{+}>\mathrm{Mg}^{2+}$. Among the cropping systems, the ion concentrations in the soil solution was higher in the continuous crop under conventional tillage, followed by the continuous crop under no-tillage, and finally by the integrated crop-livestock system and the continuous pasture. At $150 \mathrm{~cm}$ depth, the ion concentrations in soil solutions under continuous pasture and integrated crop-livestock system were always low, which indicates a low leaching risk.
\end{abstract}

Index terms: Cerrado, crop-livestock system, leaching, pasture, soil.

\section{Introdução}

Apesar de os solos do Cerrado terem sido considerados por muito tempo como terras improdutivas (Goedert, 1983), a região tem sido explorada de forma intensiva desde os primórdios dos anos 1970 e, atualmente, é uma das áreas mais importantes do país na produção de grãos e de carne. Esses solos são originalmente ácidos e pobres em nutrientes essenciais para as plantas (Sousa \& Lobato, 2004; Vendrame et al., 2010) e necessitam do uso de fertilizantes químicos e de adubos orgânicos para viabilizar a produção das culturas.

Sistemas integrados de produção que permitam utilizar mais eficientemente o solo, tanto no espaço quanto no tempo, terão papel fundamental no aumento da produção de grãos, fibras e cereais sem que novas áreas sejam incorporadas ao processo produtivo. Apesar de ainda haver espaço para melhorias, nos últimos anos tem sido observado aumento na eficiência do uso de insumos, com uma concomitante redução nas perdas por

Pesq. agropec. bras., Brasília, v.46, n.10, p.1291-1300, out. 2011 
erosão, lixiviação e volatilização (Hanson et al., 2007; Lal, 2009). De acordo com Goulding et al. (2008), uma melhor gestão dos nutrientes essenciais para as plantas é necessária, para promover a agricultura sustentável.

Um dos problemas que ocorrem, em razão do uso intensivo de fertilizantes em sistemas de cultivo, é a lixiviação, fenômeno que envolve complexa interação entre hidrologia do solo, absorção de água e nutrientes pelas plantas, e práticas de manejo (Van Es et al., 2006). Os íons solúveis, provenientes de corretivos e fertilizantes ou da decomposição da matéria orgânica, estão potencialmente disponíveis às plantas. Em períodos de alta intensidade de chuva, pode haver drenagem do excesso de água, o que favorece a movimentação descendente desses íons. Essa lixiviação é motivo de constante preocupação (Santos et al., 2002) e exige monitoramento constante, para evitar perda de nutrientes e poluição ambiental.

Os sistemas de plantio direto e de integração lavoura-pecuária são apontados como alternativas para minimizar essas perdas (Macedo, 2001). Os sistemas de cultivo convencionais, associados às práticas de revolvimento do solo para o preparo, podem acelerar a mineralização da matéria orgânica, enquanto que o não revolvimento do solo favorece o acúmulo da palhada e causa o retardamento da mineralização da matéria orgânica (MO). Ademais, a utilização de plantas de cobertura, em consórcio ou na entresafra, permite o melhor aproveitamento da água e dos nutrientes não utilizados pelas culturas principais (Anderson et al., 2001; Pacheco et al., 2011), o que evita que esses nutrientes estejam passíveis de lixiviação. Espera-se que, em sistema de integração lavoura-pecuária, haja diminuição da perda dos nutrientes por lixiviação, devido à maior possibilidade de ciclagem dos nutrientes.

O objetivo deste trabalho foi avaliar as concentrações de íons na solução de um Latossolo Vermelho, em diferentes sistemas de manejo, inclusive os de lavoura e pastagem contínua, e os rotacionados em integração lavoura-pecuária.

\section{Material e Métodos}

O trabalho foi realizado na área experimental da Embrapa Cerrados, em Planaltina, DF, em um experimento implantado em 1991 para avaliar sistemas de integração lavoura-pecuária (15³9'84"S e $47^{\circ} 44^{\prime} 41^{\prime \prime} \mathrm{W}$, altitude de $\left.1.200 \mathrm{~m}\right)$. O tipo climático, conforme classificação de Köppen, é Aw, caracterizado por duas estações bem definidas (seca e chuvosa) e ocorrência de períodos de estiagem durante a estação chuvosa (veranicos).

Os tratamentos avaliados foram dispostos em blocos ao acaso, com duas repetições em parcelas subdivididas, em uma área total de 12,8 ha. Nas parcelas, foram avaliados os seguintes sistemas de uso da terra: pastagem contínua de gramínea pura $(\mathrm{P})$; pastagem consorciada contínua (P2); rotação lavoura/pastagem consorciada (LP); rotação de pastagem consorciada/ lavoura (PL); e lavoura contínua (L). Nos sistemas LP e PL, as parcelas englobaram uma combinação fatorial de sistemas e preparo de solo: preparo convencional (PC) e sistema de plantio direto (PD). Nas subparcelas, foram avaliados dois níveis de fertilidade, F1 e F2. No sistema L, também foram utilizados os dois níveis de preparo e fertilidade, contudo, para o preparo, o arranjo foi feito em faixas horizontais, para facilitar o uso dos implementos e, nas faixas perpendiculares, os níveis de fertilidade. A área de cada subparcela foi de $4.000 \mathrm{~m}^{2}$. Nas pastagens, o método de pastejo foi o rotacionado, com ajuste da oferta de forragem (10 a $12 \mathrm{~kg}$ de matéria seca total por $100 \mathrm{~kg}$ de peso vivo). A rotação foi feita entre blocos e dentro do mesmo nível de fertilidade, não tendo havido repetição para animais. A categoria de animais utilizada foi variável: em 2005, foram utilizadas novilhas em recria; e em 2006, vacas em lactação. Informações adicionais sobre a área experimental podem ser consultadas em Marchão et al. (2009).

O solo da área experimental foi classificado como Latossolo Vermelho (LV) (Santos et al., 2006), de textura argilosa, com média de $634 \mathrm{~g} \mathrm{~kg}^{-1}$ de argila. A mineralogia da fração argila e silte compunha-se de gibsita $(50 \%)$, goethita $(18 \%)$, caulinita $(14 \%)$, hematita (7\%), quartzo e outros minerais (10\%) (Marchão et al., 2009). As características químicas do solo, nos diferentes sistemas, foram obtidas em amostras de solo, compostas de 20 subamostras, coletadas em março de 2005 nas camadas de 0 a $20 \mathrm{~cm}$ (Tabela 1).

Nas áreas com plantio convencional, o preparo do solo foi efetuado com uma gradagem pesada, após a colheita, e uma gradagem pesada seguida por uma gradagem de nivelamento, imediatamente antes do plantio. O sistema de plantio direto foi estabelecido a 
partir da safra de 1995/1996, com dessecação prévia das plantas daninhas e semeadura direta na palha. Nas áreas de pastagem contínua, o preparo convencional foi efetuado apenas na implantação, em 1991, e na renovação das pastagens, em 2000. No sistema de manejo PL, o preparo ocorreu na fase de lavoura e antes do estabelecimento das pastagens. Na safra de 2003/2004, foi semeada Urochloa brizantha como planta de cobertura, apenas nas áreas de cultivo de LP-PD, com o objetivo de formar cobertura morta para a semeadura direta do ano seguinte, de acordo com as recomendações técnicas descritas para o sistema Santa Fé de formação de pastagens ou integração lavoura-pecuária, para a região (Cobucci \& Portela, 2003).

Os herbicidas foram aplicados conforme recomendações técnicas para cada cultura. Nos anos de cultivo com a cultura da soja, foram utilizados os herbicidas trifluralin e imazaquin, em preparo convencional, em aplicação pré-emergência, e o glifosato para dessecação da vegetação de cobertura, quando se adotou o sistema de semeadura direta. Para os dois sistemas de manejo, e conforme a necessidade, foram utilizados os herbicidas: haloxyfop-methyl, fluazifop-p-butyl + fomesafen, fluazifop-p-butyl, metolachlor, diclosulam ou chlorimuron-ethyl, para controle de plantas daninhas específicas. No sistema de manejo LP, foram aplicadas doses reduzidas de haloxyfop-methyl, para controlar o crescimento de $U$. brizantha, de forma a reduzir a competição com a soja. Nas pastagens, não se utilizaram herbicidas para o manejo das plantas daninhas.

O histórico das áreas com a sequência de culturas e as aplicações totais de calcário e fertilizantes, em cada sistema de cultivo, estão descritos na Tabela 2. Nas áreas de pastagem contínua, as adubações foram feitas de acordo com as recomendações específicas, determinadas com base nas espécies cultivadas, nos resultados das análises químicas do solo e na produtividade esperada. Nas áreas de manejo PL-PD, a adubação residual das lavouras permitiu o estabelecimento das pastagens. Na safra 2004/2005, foram aplicados $10 \mathrm{~kg} \mathrm{ha}^{-1}$ de N, $100 \mathrm{~kg} \mathrm{ha}^{-1}$ de $\mathrm{P}_{2} \mathrm{O}_{5}$ e $90 \mathrm{~kg} \mathrm{ha}^{-1}$ de $\mathrm{K}_{2} \mathrm{O}$ à cultivar de soja Emgopa 313: metade no sulco de plantio em 22/12/2004 e metade a lanço na superfície, uma semana antes da semeadura. $\mathrm{Na}$ safra 2005/2006, sob as mesmas condições descritas anteriormente, foram aplicados, antes do plantio, a lanço, $175 \mathrm{~kg} \mathrm{ha}^{-1}$ da fórmula NPK 02-20-20 + micronutrientes. No sulco de plantio

Tabela 1. Resultados de análises químicas do solo, à profundidade de 0-20 cm, nos sistemas lavoura contínua, pastagem contínua e integração lavoura-pecuária ${ }^{(1)}$.

\begin{tabular}{|c|c|c|c|c|c|c|c|c|c|}
\hline Sistema $^{(2)}$ & $\begin{array}{c}\mathrm{pH} \text { em } \\
\text { água }\end{array}$ & $\begin{array}{c}\mathrm{MO} \\
\left(\mathrm{g} \mathrm{kg}^{-1}\right)\end{array}$ & P & K & Al & $\begin{array}{c}\mathrm{Ca} \\
---(\mathrm{c}\end{array}$ & $\mathrm{Mg}$ & CTC & $\begin{array}{c}\mathrm{V} \\
(\%)\end{array}$ \\
\hline L-PC & 5,5 & 29,4 & 4,7 & 63 & 0,03 & 1,85 & 0,39 & 6,13 & 39,2 \\
\hline L-PD & 5,9 & 26,1 & 6,4 & 85 & 0,00 & 1,99 & 0,54 & 6,60 & 41,6 \\
\hline $\mathrm{P}$ & 6,0 & 29,3 & 1,9 & 39 & 0,02 & 1,70 & 0,55 & 6,24 & 37,7 \\
\hline LP-PD & 6,0 & 31,2 & 4,5 & 30 & 0,00 & 2,40 & 0,67 & 7,08 & 44,1 \\
\hline PL-PD & 5,8 & 27,2 & 2,4 & 46 & 0,01 & 1,42 & 0,48 & 5,48 & 37,0 \\
\hline
\end{tabular}

${ }^{(1)}$ Os resultados são a média de duas repetições, e cada amostra foi composta de vinte subamostras. ${ }^{(2)} \mathrm{L}-\mathrm{PC}$, lavoura contínua, preparo convencional; L-PD, lavoura contínua, plantio direto; P, pastagem contínua, preparo convencional; LP-PD, rotação lavoura/pastagem, plantio direto; PL-PD, rotação pastagem/ lavoura, plantio direto; CTC, capacidade de troca catiônica; V, saturação por bases.

Tabela 2. Histórico, ciclo de cultivo e manejo da fertilidade do solo nos tratamentos ${ }^{(1)}$.

\begin{tabular}{|c|c|c|c|c|c|c|c|c|}
\hline \multirow[t]{2}{*}{ Sistema } & \multicolumn{3}{|c|}{ Ciclo de cultivo } & \multirow{2}{*}{$\begin{array}{l}\text { Calagem } \\
\left(\mathrm{Mg} \mathrm{ha}^{-1}\right)\end{array}$} & \multicolumn{3}{|c|}{ Adubação $\left(\mathrm{kg} \mathrm{ha}^{-1}\right)$} & \multirow{2}{*}{$\begin{array}{c}\text { Gesso } \\
\left(\mathrm{Mg} \mathrm{ha}^{-1}\right)\end{array}$} \\
\hline & 1991-1994 & 1995-1998 & $1999-2005$ & & $\mathrm{~N}$ & $\mathrm{P}_{2} \mathrm{O}_{5}$ & $\mathrm{~K}_{2} \mathrm{O}$ & \\
\hline L-PC & $\mathrm{S} / \mathrm{S} / \mathrm{M} / \mathrm{S}$ & $\mathrm{M} / \mathrm{S} / \mathrm{M} / \mathrm{S}$ & $\mathrm{S} / \mathrm{Mi} / \mathrm{S} / \mathrm{Mi} / \mathrm{S} / \mathrm{S} / \mathrm{So}$ & 7,5 & 308 & 1294 & 1198 & 2,8 \\
\hline L-PD & $\mathrm{S} / \mathrm{S} / \mathrm{M} / \mathrm{S}$ & $\mathrm{M} / \mathrm{S} / \mathrm{M} / \mathrm{S}$ & $\mathrm{S} / \mathrm{Mi} / \mathrm{S} / \mathrm{Mi} / \mathrm{S} / \mathrm{S} / \mathrm{So}$ & 7,5 & 308 & 1294 & 1198 & 2.8 \\
\hline $\mathrm{P}$ & $\mathrm{A} / \mathrm{A} / \mathrm{A} / \mathrm{A}$ & $\mathrm{A} / \mathrm{A} / \mathrm{A} / \mathrm{A}$ & $\mathrm{A} / \mathrm{Bd} / \mathrm{Bd} / \mathrm{Bd} / \mathrm{Bd} / \mathrm{Bd} / \mathrm{Bd}$ & 6,8 & 80 & 254 & 204 & 0,0 \\
\hline LP-PD & $\mathrm{S} / \mathrm{S} / \mathrm{M} / \mathrm{S}$ & $\mathrm{A} / \mathrm{A} / \mathrm{A} / \mathrm{A}$ & $\mathrm{S} / \mathrm{Mi} / \mathrm{S} / \mathrm{Mi} / \mathrm{S}+\mathrm{B} / \mathrm{B} / \mathrm{B}$ & 7,5 & 85 & 768 & 723 & 2,8 \\
\hline PL-PD & $\mathrm{A} / \mathrm{A} / \mathrm{A} / \mathrm{A}$ & $\mathrm{M} / \mathrm{S} / \mathrm{M} / \mathrm{S}$ & $\mathrm{P} / \mathrm{P} / \mathrm{P} / \mathrm{P} / \mathrm{P} / \mathrm{S} / \mathrm{So}$ & 7,5 & 263 & 682 & 541 & 2,8 \\
\hline
\end{tabular}

${ }^{(1)}$ L, lavoura contínua; LP, lavoura-pastagem-lavoura; PL, pastagem-lavoura-pastagem; P, pastagem contínua; A, capim-andropógon (Andropogon gayanus); B, braquiarão (Urochloa brizantha), apenas na semeadura direta; Bd, capim-braquiária (U. decumbens); M, milho (Zea mays); Mi, milheto (Pennisetum americanum); P, capim-tanzânia (Panicum maximum cv. Tanzânia); S, soja (Glycine max); So, sorgo (Sorghum bicolor). 
da cultivar de sorgo BR 304, foram aplicados, em 25/1/2006, $4 \mathrm{~kg} \mathrm{ha}^{-1}$ de $\mathrm{N}, 36 \mathrm{~kg} \mathrm{ha}^{-1}$ de $^{\mathrm{P}_{2} \mathrm{O}_{5}}$ e de $\mathrm{K}_{2} \mathrm{O}$.

No início da estação chuvosa, após a implantação da cultura anual, 60 extratores foram instalados para coletar as amostras da solução do solo às profundidades de 20 e $150 \mathrm{~cm}$, com seis repetições para cada profundidade, nos cinco sistemas selecionados. As microparcelas onde foram instalados os extratores foram cercadas, para impedir a entrada de animais e evitar a deposição de urina e fezes na área de coleta. Os extratores de solução foram construídos com tubos de PVC com $5 \mathrm{~cm}$ de diâmetro; na extremidade inferior, foi afixada uma cerâmica porosa modelo B01M3 (Soil Moisture Equipment Corp, Santa Bárbara, CA, EUA) e, na extremidade superior, foram fixadas duas mangueiras de silicone, uma para sucção e outra para retirada da solução de solo, conforme Grossmann \& Udluft (1991).

As cápsulas foram lavadas com utilização de vácuo, em solução de $\mathrm{HCl} 0,001 \mathrm{~mol} \mathrm{~L}^{-1}$ e, em seguida, três vezes com água deionizada, para eliminar impurezas. Depois de serem lavadas, as cápsulas foram mantidas imersas em água deionizada até o momento da instalação no campo. Segundo Silva et al. (2004), esses procedimentos adotados têm por objetivo eliminar interferências nas medidas da solução extraída.

Nos dois anos de coleta, observou-se que as duas estações chuvosas foram bastante diferentes, com precipitação de $1.328 \mathrm{~mm}$, em 2004-2005, e $906 \mathrm{~mm}$, em 2005-2006 (Figura 1). As chuvas foram também

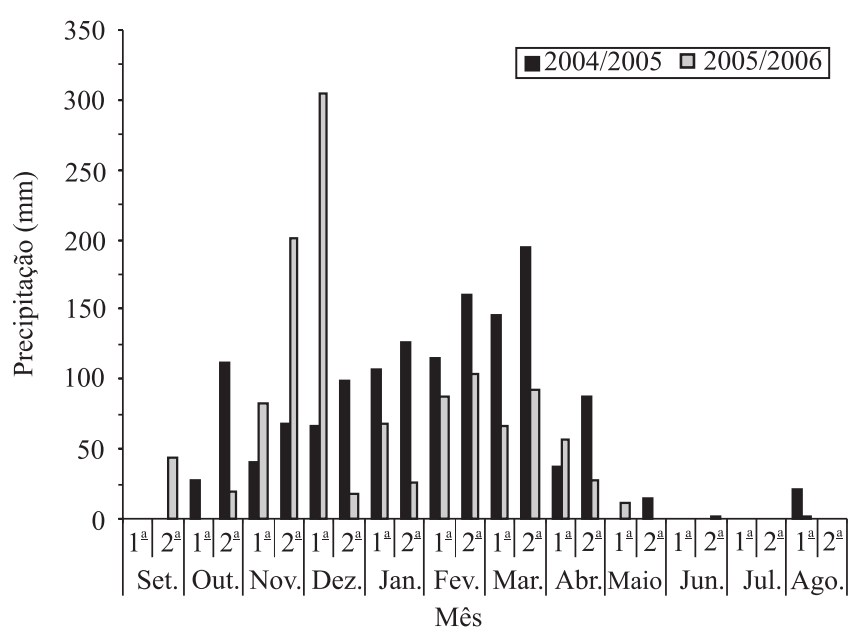

Figura 1. Precipitação pluvial quinzenal, durante o período de condução do experimento. mais irregulares na safra 2005/2006, com precipitações elevadas em dezembro e um veranico de 23 dias em janeiro. O baixo índice pluviométrico e a grande absorção de água pelas raízes, em alguns sistemas de cultivo, principalmente no tratamento PL, impediram a extração da solução do solo em algumas datas de coleta. As extrações das soluções do solo foram realizadas em $6 / 1,20 / 1,3 / 2,1 / 3,18 / 4$ e $27 / 4$, na safra $2004 / 2005$, e em $8 / 2,15 / 2,23 / 2,10 / 3,17 / 3$ e $30 / 3$, na safra 2005/2006.

Após as coletas, as amostras de solução do solo foram acondicionadas em frascos de vidro hermeticamente fechados e armazenadas em refrigerador, até o momento da realização das análises. As concentrações dos íons $\mathrm{Cl}^{-}, \mathrm{SO}_{4}{ }^{2-} \mathrm{e} \mathrm{NO}_{3}{ }^{-}$foram determinadas por cromatografia iônica, a do $\mathrm{K}^{+}$, por espectrofotometria de emissão, e as de $\mathrm{P}, \mathrm{Mg}^{2+} \mathrm{e} \mathrm{Ca}^{2+}$, por espectrofotometria de emissão atômica com fonte de plasma (ICP-AES).

Para avaliar os efeitos dos sistemas de uso sobre as concentrações dos elementos minerais, a análise dos dados foi realizada por meio do procedimento GLM (general linear models) do SAS (SAS Institute, 2008), para cada profundidade e para cada data de amostragem das soluções. A relação entre a soma dos ânions, ou concentração do nitrato, e a soma dos cátions foi calculada por análise de regressão linear, com uso do aplicativo Excel da Microsoft.

\section{Resultados e Discussão}

As concentrações de nitrato na solução do solo apresentaram diferenças significativas entre os sistemas de cultivo. Durante o ano agrícola 2004/2005, na área de lavoura contínua em preparo convencional (L-PC), a concentração do nitrato na solução do solo foi maior do que nos outros sistemas, nas duas profundidades avaliadas (Figuras 2 A e B). Na primeira coleta, as concentrações foram de $8.500 \mu \mathrm{mol} \mathrm{L}^{-1}$, à profundidade de $20 \mathrm{~cm}$, e $600 \mu \mathrm{mol} \mathrm{L}-1$ à profundidade de $150 \mathrm{~cm}$. Essas concentrações são maiores que as observadas por Moraes (1991) e Lilienfein et al. (2000) em sistema de manejo similares, que apresentaram resultados inferiores a $3.000 \mu \mathrm{mol} \mathrm{L}{ }^{-1}$, nos horizontes superficiais, e $100 \mu \mathrm{mol} \mathrm{L}^{-1} \mathrm{a} 100 \mathrm{~cm}$. Contudo, após a terceira coleta, as concentrações foram reduzidas a zero e $200 \mu \mathrm{mol} \mathrm{L} \mathrm{L}^{-1}$ às profundidades de $20 \mathrm{e}$ de $150 \mathrm{~cm}$, respectivamente. Segundo Alcântara \& Camargo (2010), há baixa afinidade entre o nitrato e os sítios de adsorção dos 
solos; assim, a rápida diminuição das concentrações após a primeira coleta, principalmmente a $20 \mathrm{~cm}$ de profundidade, pode estar relacionada à absorção do nitrato pelas raízes. O sistema lavoura contínua, em plantio direto (L-PD), também apresentou concentrações de nitrato significativamente maiores do que as dos sistemas integrados com pastagens, com concentrações iniciais que alcançaram $2.700 \mu \mathrm{mol} \mathrm{L}^{-1}$ a $20 \mathrm{~cm}$ e $200 \mu \mathrm{mol} \mathrm{L}{ }^{-1}$ a $150 \mathrm{~cm}$. Essas concentrações são similares às observadas por Lilienfein et al. (2000). Segundo Bayer \& Mielniczuk (1997), a menor concentração de nitrogênio, no sistema de semeadura em plantio direto, pode ser atribuída à menor taxa de decomposição da matéria orgânica e dos resíduos culturais incorporados ao solo. As áreas de pastagem contínua $(\mathrm{P})$ e rotações lavoura/pastagem (LP-PD) apresentaram valores próximos a zero, nas duas profundidades, durante todo o período de avaliação.

No ano agrícola 2005/2006, as concentrações do nitrato na solução do solo, nos diferentes sistemas avaliados (L-PC, L-PD, P, LP-PD e PL-PD), foram menores do que as registradas no ano agrícola anterior (Figura $2 \mathrm{C}$ e D). Para a profundidade de $20 \mathrm{~cm}$, sob os sistemas de lavoura (L-PC, L-PD, LP-PD), foram observadas concentrações de nitrato de 500 a $3.000 \mu \mathrm{mol} \mathrm{L}{ }^{-1}$. Nas áreas sob pastagem (P e PL-PD), as concentrações foram significativamente menores, sempre abaixo de $100 \mu \mathrm{mol} \mathrm{L}-1$ (Figura $2 \mathrm{C}$ ). À profundidade de $150 \mathrm{~cm}$, o tratamento L-PC
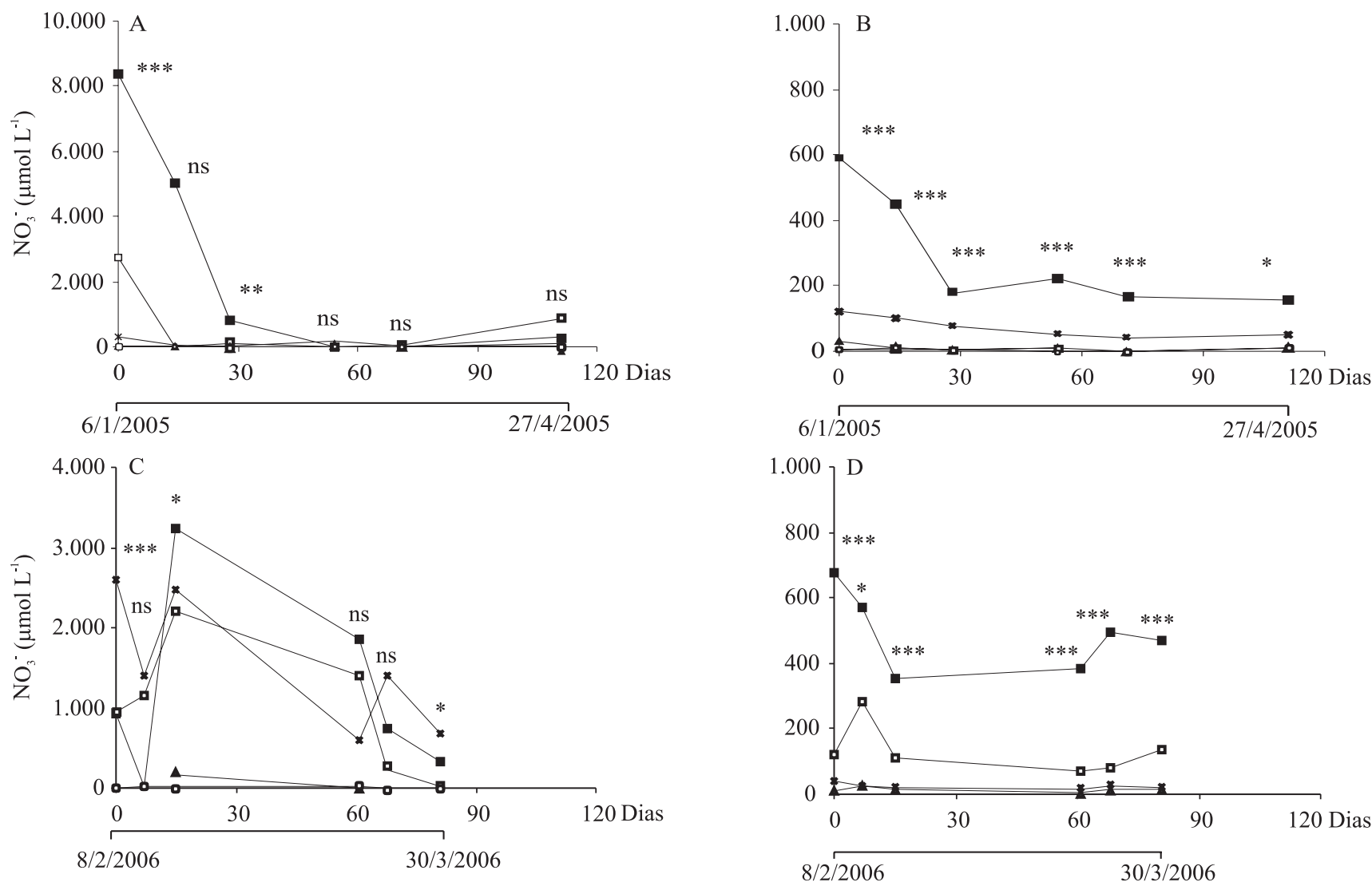

Data

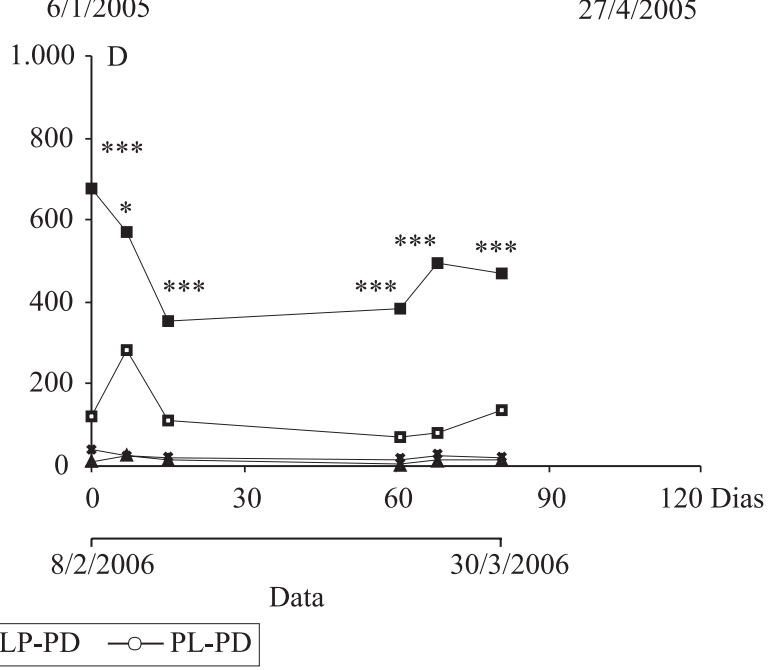

Figura 2. Concentrações de nitrato na solução do solo, às profundidades de 20 (A e C) e $150 \mathrm{~cm}(\mathrm{~B}$ e D), durante os anos agrícolas de 2004/20005 (A e B) e 2005/2006 (C e D), sob cinco sistemas de cultivo. L-PC, lavoura contínua, preparo convencional; L-PD, lavoura contínua, plantio direto; P, pastagem contínua, preparo convencional; LP-PD, rotação lavoura/

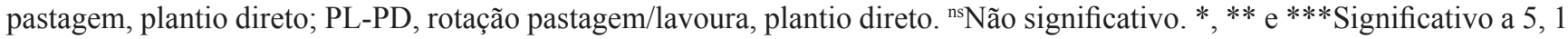
e $0,1 \%$ de probabilidade, respectivamente, para cada data de coleta das soluções do solo. 
destacou-se dos demais, com concentrações de nitrato de 400 a $700 \mu \mathrm{mol} \mathrm{L}^{-1}$, seguidas pelo tratamento L-PD (Figura 2 D). As concentrações de nitrato nas áreas sob LP-PD e pastagem contínua apresentaram valores próximos a zero, como no ano anterior. A área sob PL-PD não foi avaliada no ano 2005/2006, em razão da baixa umidade ocorrida no período, que impossibilitou a coleta de amostra da solução do solo.

As concentrações dos cátions $\mathrm{K}^{+}, \mathrm{Mg}^{2+}$ e $\mathrm{Ca}^{2+}$ na solução de solo foram apresentadas apenas para a safra 2004/2005 (Figura 3). Elas seguiram a mesma tendência observada para o nitrato. As concentrações de $\mathrm{K}^{+}, \mathrm{Mg}^{2+}$ e $\mathrm{Ca}^{2+}$ na solução do solo, entre os sistemas de cultivo, foram também significativamente diferentes para as profundidades e as datas de coleta, exceto à profundidade de $20 \mathrm{~cm}$, na quarta coleta, para $\mathrm{Ca}^{2+} \mathrm{e}$ $\mathrm{Mg}^{2+}$, e na quarta e quinta coletas para $\mathrm{K}^{+}$.

À profundidade de $20 \mathrm{~cm}$, na safra de 2004/2005, foi possível observar um rápido decréscimo das concentrações de $\mathrm{K}^{+}$, que passaram de $1.800 \mu \mathrm{mol} \mathrm{L}^{-1}$, no tratamento L-PC, no início de plantio, a $65 \mu \mathrm{mol} \mathrm{\textrm {L } ^ { - 1 }}$ no final da estação chuvosa (Figura $3 \mathrm{~A}$ ). Assim como ocorreu com o nitrato, as concentrações de $\mathrm{K}^{+}$na solução do solo foram mais elevadas no tratamento L-PC (lavoura com preparo convencional) do que nos demais. Esse resultado diverge dos apresentados por Lilienfein et al. (2000), que observaram maiores concentrações de $\mathrm{K}^{+}$na solução do solo, em áreas sob plantio direito. As áreas sob pastagem (P) também apresentaram concentrações muito baixas de $\mathrm{K}^{+}$na solução do solo. Em relação a esse resultado, deve-se ressaltar que a última adubação potássica $\left(60 \mathrm{~kg} \mathrm{ha}^{-1} \mathrm{de}_{2} \mathrm{O}\right)$ realizada na pastagem foi em 2002. À profundidade de $150 \mathrm{~cm}$, as concentrações não variaram muito no período de coleta (Figura 3 B), e não houve diferença entre tratamentos, exceto para o L-PD. As concentrações de $\mathrm{K}^{+}$na solução do solo, observadas nas áreas sob cultivo contínuo, confirmam as obtidas por Espinoza Garrido \& Reis (1984), Moraes (1991) e Miranda et al. (2006). A menor concentração foi encontrada na área sob pastagem contínua (P), seguida dos tratamentos sob integração lavoura-pecuária (LP-PD e PL-PD).

As concentrações de magnésio na solução do solo foram menores que as de $\mathrm{K}^{+}$(Figuras $3 \mathrm{C}$ e $\mathrm{D}$ ), no entanto, seguiram um padrão semelhante. A $20 \mathrm{~cm}$ de profundidade, observou-se rápido decréscimo das concentrações, e todos os tratamentos mostraram o mesmo comportamento (Figura $3 \mathrm{C}$ ). A $150 \mathrm{~cm}$, embora não tenha ocorrido grande diferença entre os tratamentos, o solo sob lavoura contínua (L-PC) apresentou concentrações maiores de $\mathrm{Mg}^{2+}$ na solução do solo (Figura 3 D).

Quanto às concentrações de $\mathrm{Ca}^{2+}$ na solução do solo, houve variações similares às observadas com potássio e magnésio, em todos os tratamentos. À profundidade de $20 \mathrm{~cm}$, houve rápido decréscimo das concentrações da primeira para a última coleta (Figura $3 \mathrm{E}$ ). À profundidade de $150 \mathrm{~cm}$, as concentrações de $\mathrm{Ca}^{2+}$ também não variaram entre os períodos de coleta. $\mathrm{O}$ tratamento L-PC apresentou concentrações significativamente maiores do que os demais tratamentos (Figura $3 \mathrm{~F}$ ).

As concentrações de cloro, sulfato e fosfato na solução do solo (Tabela 3 ) foram muito menores do que as observadas para $\mathrm{NO}_{3}{ }^{-}, \mathrm{K}^{+}, \mathrm{Mg}^{2+} \mathrm{e} \mathrm{Ca}^{2+}$ (Figuras $2 \mathrm{e} 3$ ). As concentrações de cloreto foram significativamente maiores no início das estações chuvosas, à profundidade de $20 \mathrm{~cm}$, principalmente nos tratamentos L-PC e LP-PD. Entretanto, à profundidade de $150 \mathrm{~cm}$, não se observaram grandes variações entre os tratamentos, com concentrações médias de $200 \mu \mathrm{mol} \mathrm{L}^{-1}$, exceto para o tratamento $\mathrm{P}$, em que a concentração média foi de $70 \mu \mathrm{mol} \mathrm{L}{ }^{-1}$. As concentrações mais elevadas de cloreto, nas áreas de lavoura contínua, estão relacionadas à adubação potássica, em que se aplicou o fertilizante cloreto de potássio, enquanto nas áreas sob pastagem contínua não foi feita nenhuma adubação com potássio na safra 2004/2005. De acordo com Lilienfein et al. (2000), as variações na concentração do $\mathrm{Cl}^{-}$podem estar relacionadas a variações nos teores de água, e o consequente efeito de diluição do $\mathrm{Cl}^{-}$, ou à sorção do $\mathrm{Cl}^{-}$pelos óxidos com cargas positivas, como apontam Morais et al. (1976). As concentrações de sulfato também foram muito baixas, com médias de 6 a 42 e de 1 a $13 \mu \mathrm{mol} \mathrm{L}^{-1}$ às profundidades de 20 e $150 \mathrm{~cm}$, respectivamente. Elas foram também significativamente diferentes entre os sistemas de cultivo, no início das estações chuvosas (primeira a terceira coletas). As concentrações de P ficaram sempre muito baixas $\left(<3 \mu \mathrm{mol} \mathrm{L} \mathrm{L}^{-1}\right)$. Isto está relacionado à grande afinidade desse íon com a fase sólida do solo (Eberhardt et al., 2008).

A similaridade nas variações das concentrações dos três cátions avaliados e do ânion nitrato, pode ser atribuída ao equilíbrio entre os íons. A concentração dos elementos na solução do solo é controlada por 
vários atributos físico-químicos do solo, tais como: $\mathrm{pH}$, complexação iônica e capacidade de troca catiônica. Os ânions $\mathrm{NO}_{3}^{-}, \mathrm{Cl}^{-}$e $\mathrm{SO}_{4}^{2-}$, provenientes da mineralização dos resíduos orgânicos, dos fertilizantes e da água da chuva, pouco adsorvidos pelos solos, ficam livres na solução (Oliveira et al., 2002).
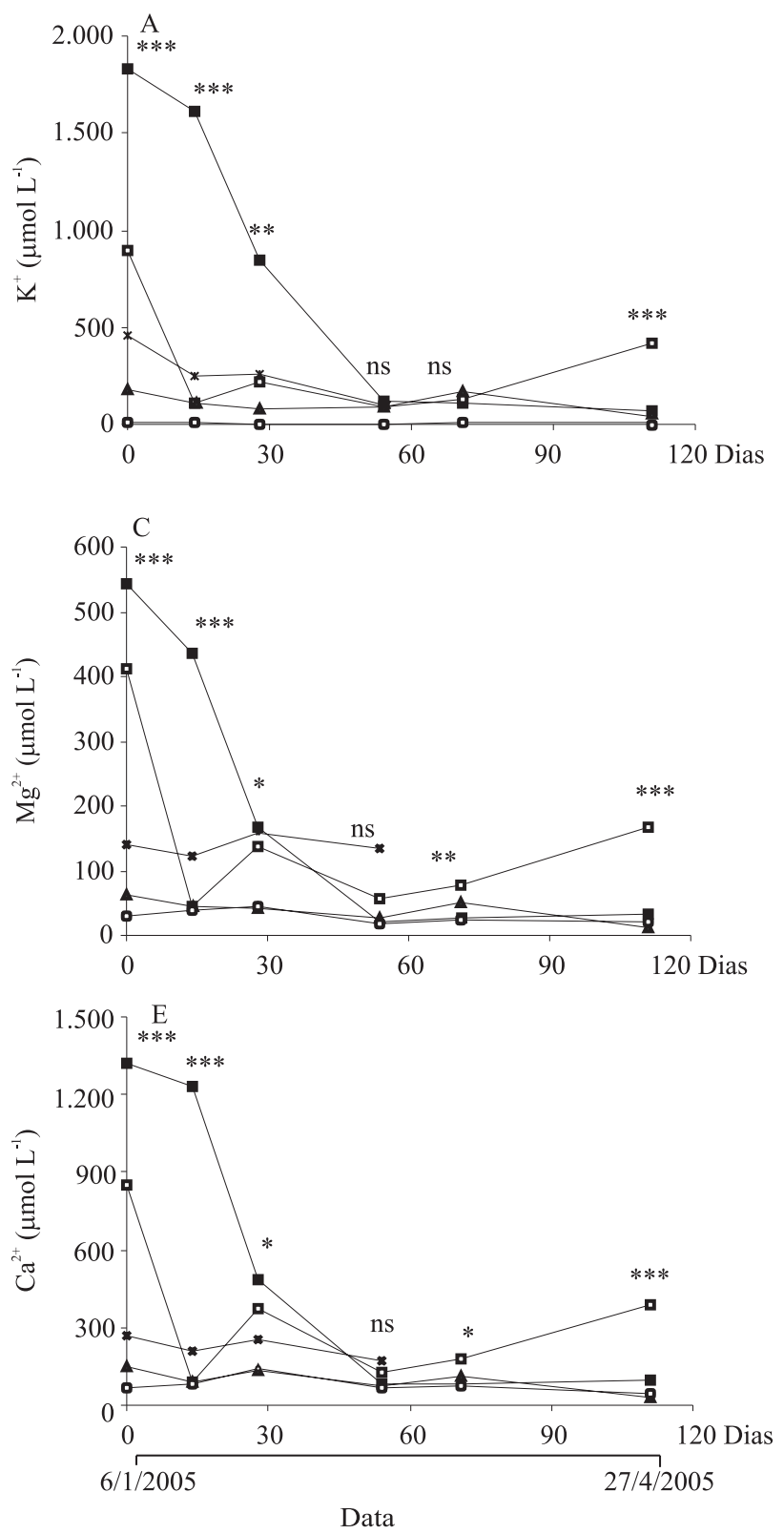

Em virtude da associação iônica na solução do solo, com a neutralização de cargas, os ânions promoveram a presença dos cátions básicos $\left(\mathrm{Ca}^{2+}, \mathrm{Mg}^{2+} \mathrm{e} \mathrm{K}{ }^{+}\right)$. Dessa forma, as perdas de $\mathrm{NO}_{3}^{-}, \mathrm{Cl}^{-}$e $\mathrm{SO}_{4}^{2-}$ por lixiviação podem contribuir também para a lixiviação dessas bases (Moraes, 1991).
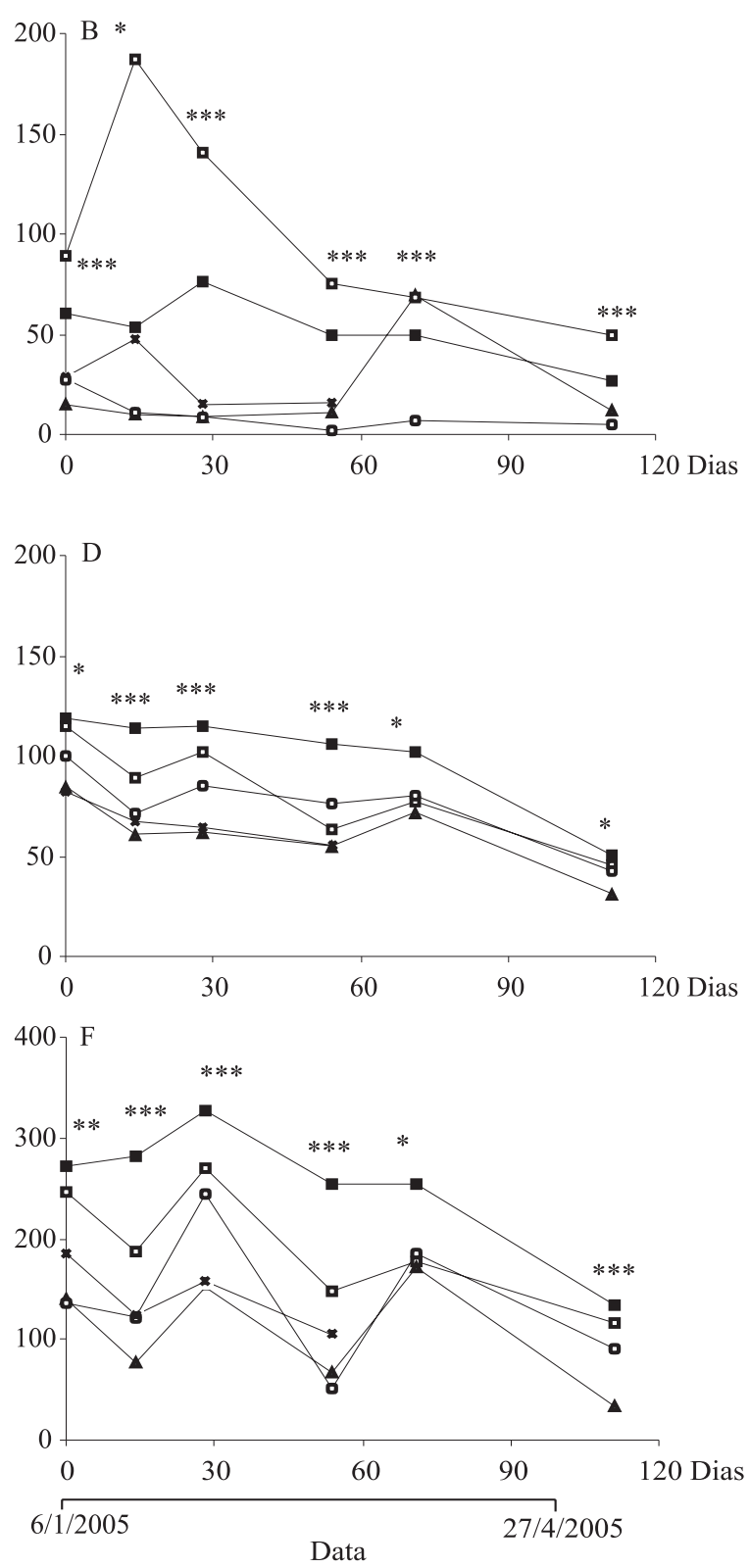

$\rightarrow \mathrm{L}-\mathrm{PC} \rightarrow \mathrm{a}-\mathrm{PD} \rightarrow \mathrm{-P} \rightarrow \mathrm{LP}-\mathrm{PD} \rightarrow \mathbf{0}-\mathrm{PL}-\mathrm{PD}$

Figura 3. Concentrações dos cátions potássio (A, B), magnésio $(C, D)$ e cálcio $(E, F)$, na solução do solo, sob diferentes sistemas de cultivo, às profundidades de 20 (A, C, E) e 150 cm (B, D, F), no ano agrícola 2004/2005. L-PC, lavoura contínua, preparo convencional; L-PD, lavoura contínua, plantio direto; P, pastagem contínua, preparo convencional; LP-PD, rotação lavoura/ pastagem, plantio direto; PL-PD, rotação pastagem/lavoura, plantio direto. (ns) Não significativo. *, ** e *** Significativo a 5, 1 e $0,1 \%$ de probabilidade, respectivamente, para cada data de coleta das soluções do solo. 
Tabela 3. Concentrações médias de cloreto, sulfato e fosfato na solução de solo $\left(\mu \mathrm{mol} \mathrm{L}^{-1}\right)$, coletadas das profundidades de 20 e 150 cm, e cálculo do balanço iônico nos anos de 2004/2005 e 2005/2006, sob cinco sistemas de cultivo.

\begin{tabular}{|c|c|c|c|c|c|c|c|c|c|c|}
\hline \multirow[t]{2}{*}{ Sistema $^{(1)}$} & \multicolumn{5}{|c|}{ À $20 \mathrm{~cm}$ de profundidade } & \multicolumn{5}{|c|}{ À $150 \mathrm{~cm}$ de profundidade } \\
\hline & $\mathrm{Cl}^{-}$ & $\mathrm{SO}_{4}^{2-}$ & $\mathrm{H}_{2} \mathrm{PO}_{4}^{-}$ & $\sum$ cátions & Eânions & $\mathrm{Cl}^{-}$ & $\mathrm{SO}_{4}{ }^{2-}$ & $\mathrm{H}_{2} \mathrm{PO}_{4}^{-}$ & Ecátions & Kânions \\
\hline & \multicolumn{10}{|c|}{ Ano agrícola 2004/2005 } \\
\hline L-PC & 246,8 & 20,8 & 2,2 & 2.272 & 2.721 & 218,3 & 5,3 & 2,2 & 763 & 525 \\
\hline L-PD & 88,6 & 42,8 & 2,0 & 1.278 & 798 & 195,6 & 13,3 & 2,3 & 647 & 299 \\
\hline $\mathrm{P}$ & 56,5 & 6,8 & 1,6 & 386 & 103 & 79,6 & 7,1 & 1,7 & 356 & 108 \\
\hline LP-PD & 218,0 & 37,5 & 3,2 & 989 & 386 & 160,5 & 1,0 & 1,8 & 447 & 171 \\
\hline \multirow[t]{2}{*}{ PL-PD } & 18,6 & 11,2 & 1,8 & 226 & 71 & 191,5 & 4,3 & 1,4 & 487 & 207 \\
\hline & \multicolumn{10}{|c|}{ Ano agrícola 2005/2006 } \\
\hline L-PC & 154,8 & 0,1 & 0,0 & 1.881 & 1.375 & 207,0 & 0,1 & 0,0 & 1.009 & 737 \\
\hline L-PD & 377,6 & 0,0 & 0,0 & 3.133 & 1.401 & 211,8 & 0,0 & 0,0 & 373 & 346 \\
\hline $\mathrm{P}$ & 131,2 & 0,1 & 0,0 & 352 & 220 & 126,7 & 0,1 & 0,0 & 180 & 141 \\
\hline LP-PD & 316,3 & 0,1 & 0,0 & 3.231 & 1.847 & 191,2 & 0,1 & 0,0 & 340 & 218 \\
\hline PL-PD & 35,0 & 0,1 & 0,0 & 178 & 44 & 196,4 & 0,2 & 0,0 & 345 & 197 \\
\hline
\end{tabular}

(1)L-PC, lavoura contínua, preparo convencional; L-PD, lavoura contínua, plantio direto; P, pastagem contínua, preparo convencional; LP-PD, rotação lavoura/pastagem, plantio direto; PL-PD, rotação pastagem/lavoura, plantio direto.

A relação entre a soma dos ânions e a soma dos cátions ( $\Sigma$ ânions $=486+0,86 \Sigma$ cátions $)$ e entre concentração do nitrato e a soma dos cátions (nitrato $=596+0,93 \Sigma$ cátions) foram altamente significativas, com coeficiente de determinação $\left(\mathrm{R}^{2}\right)$ de 0,63 e 0,62 , respectivamente. A inclinação de 0,93 observada na regressão linear indica que o aumento da concentração de nitrato induz aumento quase que diretamente proporcional da carga de cátions na solução do solo. No caso do sistema com lavoura contínua, em particular, o aumento da concentração de $\mathrm{NO}_{3}{ }^{-}$pode acarretar a movimentação correspondente de cátions no perfil do solo.

No entanto, a soma de ânions $\left(\mathrm{NO}_{3}^{-}+\mathrm{Cl}^{-}+\mathrm{SO}_{4}{ }^{2-}\right)$ na solução do solo de todos os tratamentos foi menor do que a soma de cátions $\left(\mathrm{Ca}^{2+}+\mathrm{Mg}^{2+}+\mathrm{K}^{+}\right)$, conforme Tabela 3. Isso é confirmado também pelo valor da intercessão da reta da regressão entre a soma de ânions e a soma dos cátions, que alcançou $486 \mu \mathrm{mol} \mathrm{\textrm {L } ^ { - 1 }}$ para a soma de ânions e $596 \mu \mathrm{mol} \mathrm{L}^{-1}$ para o nitrato. Moraes (1991) também observou que a soma de ânions representou de $35-40 \%$ da soma de cátions. Uma hipótese para explicar a alta quantidade de cátions na solução do solo, seria a presença do ânion bicarbonato $\left(\mathrm{HCO}_{3}^{-}\right)$na solução.

A concentração de bicarbonato é regulada pela pressão de $\mathrm{CO}_{2}\left(\mathrm{P}_{\mathrm{CO}_{2}}\right)$ na atmosfera do solo, segundo a equação proposta por Reuss \& Johnson (1986): $\left[\mathrm{H}^{+}\right]$ $\left[\mathrm{HCO}_{3}^{-}\right]=\mathrm{P}_{\mathrm{CO}_{2}} \times 10^{-7,81}$. Com pH médio de 6,5 , as $\mathrm{P}_{\mathrm{CO}_{2}}$ de 0,008 e $0,07 \mathrm{~atm}$ correspondem às concentrações média e máxima do deficit de carga de 380 e $3.600 \mu \mathrm{mol} \mathrm{L}{ }^{-1}$, respectivamente. Esses valores são coerentes com os valores de $\mathrm{P}_{\mathrm{CO}_{2}}$ encontrados em solo por Buyanovsky \& Wagner (1983). O bicarbonato, que não se move no solo, ou se move muito lentamente, serviria para compensar as cargas de outros ânions, absorvidos pela planta. Nessas condições, as perdas de cátions por lixiviação, principalmente cálcio e magnésio, não podem ser eliminadas totalmente, conforme o observado nas áreas sob pastagem e de integração lavoura-pecuária.

\section{Conclusões}

1. Para as profundidades de $20 \mathrm{~cm}$ e de $150 \mathrm{~cm}$, nos diferentes sistemas avaliados, as concentrações de íons na solução do solo ocorrem na seguinte ordem decrescente: $\mathrm{NO}_{3}{ }^{-}>\mathrm{Cl}^{-}>\mathrm{SO}_{4}{ }^{2-}>\mathrm{H}_{2} \mathrm{PO}_{4}^{-}$, para os ânions; e $\mathrm{Ca}^{2+}>\mathrm{K}^{+}>\mathrm{Mg}^{2+}$, para os cátions.

2. As concentrações dos ânions e cátions na solução do solo apresentam padrão similar, e o íon nitrato apresenta as maiores concentrações.

3. Considerados os sistemas de manejo, as concentrações de íons na solução do solo, independentemente da profundidade analisada, decrescem na seguinte ordem: as de lavoura contínua sob preparo convencional, lavoura contínua sob plantio direto, integração lavoura-pecuária e pastagem contínua.

4. As concentrações dos íons na solução do solo, à profundidade de $150 \mathrm{~cm}$, sob pastagem contínua e 
integração lavoura-pecuária, são as mais baixas, o que indica menores riscos de lixiviação, em comparação às de áreas de lavoura contínua, principalmente aquelas com preparo do solo convencional; nesses sistemas, o íon nitrato apresenta maior potencial de perdas por lixiviação.

\section{Agradecimentos}

À Coordenação de Aperfeiçoamento de Pessoal de Nível Superior, pela concessão de bolsa.

\section{Referências}

ALCÂNTARA, M.A.K. de; CAMARGO, O.A. de. Movimentação de nitrato em horizonte superficial e subsuperficial de Latossolo e Nitossolo com cargas variáveis. Pesquisa Agropecuária Brasileira, v.45, p.81-88, 2010.

ANDERSON, S.; GÜENDEL, S.; POUND, B.; TRIOMPHE, B. Cover crops in smallholder agriculture: lessons from Latin America. London: IT Publications, 2001. 136p.

BAYER, C.; MIELNICZUK, J. Características químicas do solo afetadas por métodos de preparo e sistemas de cultura. Revista Brasileira de Ciência do Solo, v.21, p.105-112, 1997.

BUYANOVSKY, G.A.; WAGNER, G.H. Annual cycles of carbon dioxide level in soil air. Soil Science Society of America Journal, v.47, p.1139-1145, 1983.

COBUCCI, T.; PORTELA, C.M. de O. Manejo de herbicidas no sistema Santa Fé e na braquiária como fonte de cobertura morta. In: KLUTHCOUSKI, J.; STONE, L.F.; AIDAR, H. (Ed.). Integração lavoura pecuária. Santo Antônio de Goiás: Embrapa Arroz e Feijão, 2003. p.443-458.

EBERHARDT, D.N.; VENDRAME, P.R.S.; BECQUER ,T.; GUIMARÃES, M. de F. Influência da granulometria e da mineralogia sobre a retenção do fósforo em Latossolos sob pastagens no Cerrado. Revista Brasileira de Ciência do Solo, v.32, p.1009-1016, 2008.

ESPINOZA GARRIDO, W.; REIS, A.E.G. dos. Lixiviação em Latossolo Vermelho-Escuro de Cerrado. II. Magnitude e variabilidade de fenômenos sob irrigação. Pesquisa Agropecuária Brasileira, v.19, p.85-94, 1984.

GOEDERT, W.J. Management of the Cerrado soils of Brazil: a review. Journal of Soil Science, v.34, p.405-428, 1983.

GOULDING, K.; JARVIS, S.; WHITMORE, A. Optimizing nutrient management for farm systems. Philosophical Transactions of the Royal Society B: Biological Sciences, v.363, p.667-680, 2008.

GROSSMANN, J.; UDLUFT, P. The extraction of soil water by the suction-cup method: a review. Journal of Soil Science, v.42, p.83-93, 1991.

HANSON, J.D.; LIEBIG, M.A.; MERRILL, S.D.; TANAKA, D.L.; KRUPINSKY, J.M.; STOTT, D.E. Dynamic cropping systems: increasing adaptability amid an uncertain future. Agronomy Journal, v.99, p.939-943, 2007.

LAL, R. Soils and food sufficiency: a review. Agronomy for Sustainable Development, v.29, p.113-133, 2009.

LILIENFEIN, J.; WILCKE, W.; LIMA, S.C.; VILELA, L.; THOMAS, R.; ZECH, W. Nutrient concentrations in soil solution of some Brazilian Oxisols under conventional and no-tillage systems in the early part of the rainy season. Australian Journal of Soil Research, v.38, p.851-866, 2000.

MACEDO, M.C.M. Integração lavoura e pecuária: alternativa para sustentabilidade da produção animal. In: SIMPÓSIO SOBRE MANEJO DA PASTAGEM, 18., 2001, Piracicaba. Anais. Piracicaba: Universidade de São Paulo, 2001. p.257-283.

MARCHÃO, R.L.; BECQUER, T.; BRUNET, D.; BALBINO, L.C.; VILELA. L.; BROSSARD, M. Carbon and nitrogen stocks in a Brazilian clayey Oxisol: 13-year effects of integrated crop-livestock management systems. Soil \& Tillage Research, v.103, p.442-450, 2009.

MIRANDA, J.; COSTA, L.M. da; RUIZH, A.; EINLOFT, R. Composição química da solução de solo sob diferentes coberturas vegetais e análise de carbono orgânico solúvel no deflúvio de pequenos cursos de água. Revista Brasileira de Ciência do Solo, v.30, p.633-647, 2006.

MORAES, J.F.V. Movimento de nutrientes em Latossolo Vermelho-Escuro. Pesquisa Agropecuária Brasileira, v.30, p.85-97, 1991.

MORAIS, F.I.; PAGE, A.L.; LUND, L.J. The effect of pH, salt concentration, and nature of electrolytes on the charge characteristics of Brazilian tropical soils. Soil Science Society of America Journal, v.40, p.521-527, 1976.

OLIVEIRA, F.H.T.; NOVAIS, R.F.; ALVAREZ V., V.H.; CANTARUTTI, R.B.; BARROS, N.F. Fertilidade do solo no sistema plantio direto. In: ALVAREZ V., V.H.; SCHAEFER, C.E.G.R.; BARROS, N.F. de; MELLO, J.W.V. de; COSTA, L.M. da. (Ed.). Tópicos em ciência do solo. Viçosa: Sociedade Brasileira de Ciência do Solo, 2002. v.2, p.393-486.

PACHECO, L.P.; LEANDRO, W.M.; MACHADO, P.L.O. de A.; ASSIS, R.L. de; COBUCCI, T.; MADARI, B.E.; PETTER, F.A. Produção de fitomassa e acúmulo e liberação de nutrientes por plantas de cobertura na safrinha. Pesquisa Agropecuária Brasileira, v.46, p.17-25, 2011.

REUSS, J.O.; JOHNSON, D.W. Acid deposition and the acidification of soil and waters. New York: Springer-Verlag, 1986. 119p.

SANTOS, A.B. dos; FAGERIA, N.K.; ZIMMERMANN, F.J.P. Atributos químicos do solo afetado pelo manejo da água e do fertilizante potássico na cultura de arroz irrigado. Revista Brasileira de Engenharia Agrícola e Ambiental, v.6, p.12-16, 2002.

SANTOS, H.G. dos; JACOMINE, P.K.T.; ANJOS, L.H.C. dos; OLIVEIRA, V.A. de; OLIVEIRA, J.B. de; COELHO, M.R.; LUMBRERAS, J.F.; CUNHA, T.J.F. (Ed.). Sistema brasileiro de classificação de solos. 2.ed. Rio de Janeiro: Embrapa Solos, 2006. $306 \mathrm{p}$.

SAS INSTITUTE. SAS/STAT User's guide. Version 9.2. Cary: SAS Institute, 2008. 
SILVA, R.H.; ROSOLEM, C.A.; WONG, J. Liberação de cálcio e magnésio por cápsulas porosas de porcelana usadas na extração de solução do solo. Revista Brasileira de Ciência do Solo, v.28, p.605-610, 2004.

SOUSA, D.M.G. de; LOBATO, E. (Ed.). Cerrado: correção do solo e adubação. 2.ed. Brasília: Embrapa Informação Tecnológica; Planaltina: Embrapa Cerrados, 2004. 416p.
VAN ES, H.M.; SOGBEDJI, J.M.; SCHINDELBECK, R.R. Effect of manure application timing, crop, and soil type on nitrate leaching. Journal of Environmental Quality, v.35, p.670-679, 2006.

VENDRAME, P.R.S.; BRITO, O.R.; GUIMARÃES, M.F.; MARTINS, E.S.; BECQUER, T. Fertility and acidity status of Latossolos (Oxisols) under pasture in the Brazilian Cerrado. Anais da Academia Brasileira de Ciências, v.82, p.1085-1094, 2010.

Recebido em 22 de julho de 2010 e aprovado em 9 de agosto de 2011 